\section{Problems with Precision: A Response to "Comments on 'Data Mining Static Code Attributes to Learn Defect Predictors"'}

\author{
Tim Menzies, Member, IEEE, Alex Dekhtyar, \\ Justin Distefano, and Jeremy Greenwald
}

Abstract-Short abstract needed, please.

Index Terms-Defect prediction, accuracy measures, static code attributes, empirical.

\section{INTRODUCTION}

ZHANG and Zhang [15] argue that the low precision detectors seen in Menzies et al.'s paper "Data Mining Static Code Attributes to Learn Defect Predictors" [13] (hereafter, DMP) are "not satisfactory for practical purposes." They demand that "a good prediction model should achieve both high Recall and high Precision" (which we will denote as "high precision\&recall"). All other detectors, they argue, "may lead to impractical prediction models."

We have a different view, and this short note explains why. While we disagree with the Zhangs' conclusions, we find that their derived equation is an important result. The insightful feature of the Zhangs' equation is that it can use information about the problem at hand to characterize the preconditions for high precision and high recall detectors. To the best of our knowledge, no such characterization has been previously reported (at least, not in the software engineering literature).

\section{PRECISION INSTABILITY}

Precision instability is the real reason that we do not assess performance in terms of precision. But, precision instability was not discussed in the DMP paper. Hence, Zhang and Zhang are right to complain about our selection of assessment criteria.

We first detected precision instability in several NASA data sets. If researchers want to demonstrate that detector generator $A$ is better than method $B$, then they must measure the performance of $A$ and $B$ under a variety of treatments. Fig. 1 shows one such study [1], where different learners (e.g., M5', J48, ROCKY, LSR) were

- trained on one of five data sets,

- $\quad$ self-applied on the same training set (to generate a baseline performance measure), and then

- tested on each of the other four data sets.

- T. Menzies is with the Lane Department of Computer Science and Electrical Engineering, West Virginia University, Morgantown, WV 26506.E-mail: tim@menzies.us.

- A. Dekhtyar is with the University of Kentucky. E-mail: dekhtyar@cs.uky.edu.

- J. Distefano is with Integrated Software Metrics. E-mail: jdistefano@ismwv.com.

- J. Greenwald is with the Department of Computer Science, Portland State University, PO Box 751, Portland, OR 97207-0751.

E-mail: jgreen@cs.pdx.edu.

Manuscript received 21 Feb. 2007; revised 11 Mar. 2007; accepted DD Mmmm, YYYY; published online 2 July 2007.

Recommended for acceptance by M. Harman.

For information on obtaining reprints of this article, please send e-mail to: tse@computer.org, and reference IEEECS Log Number TSE-0302-1206rebuttal.

Digital Object Identifier no. 10.1109/TSE.2007.70721.
The results are shown in Fig. 1. On most performance measures, the detectors were remarkably stable and similar performances were observed in different data sets. The exception was prec (precision), which had very large standard deviations when applied to different data sets. Hence, if detectors were to be assessed in terms of precision, it would be very difficult to show that method $A$ was better than $B$.

Another problem with precision instability is maintainability. It is important to stabilize a project's defect detectors so that they remain viable after release. For example, no project manager wants to discover that their 80 percent precise detector is only 25 percent precise when a project update is pushed out three months later.

We show below that the Zhangs' equation can explain precision instability, as well as certain other prior empirical results.

\section{The Mathematics of Precision}

Let $\{A, B, C, D\}$ denote the true negatives, false negatives, false positives, and true positives (respectively) found by a binary detector. Certain standard measures can be computed from $A, B, C, D$ :

$$
\begin{array}{ll}
\text { pd }=\text { recall }= & \frac{D}{B+D}, \\
\text { pf }= & \frac{C}{A+C}, \\
\text { prec }=\text { precision }= & \frac{D}{D+C}, \\
\text { acc }=\text { accuracy }= & \frac{A+D}{A+B+C+D}, \\
\text { selectivity }= & \frac{C+D}{A+B+C+D}, \\
\text { neg/pos }= & \frac{A+C}{B+D} .
\end{array}
$$

The last measure $(n e g / p o s)$ is most important to the subsequent discussion. The Zhangs' equation is derived as follows:

$$
\text { prec }=\frac{D}{D+C}=\frac{1}{1+\frac{C}{D}}=\frac{1}{1+\text { neg } / \text { pos } \cdot p f / \text { recall }},
$$

which can be rearranged to

$$
p f=\frac{\text { pos }}{n e g} \cdot \frac{(1-\text { prec })}{\text { prec }} \cdot \text { recall } .
$$

Note that, in (2), when recall is fixed, the false alarm rate becomes controlled by precision and a fixed constant determined by the data set being examined, i.e., when $(\alpha=n e g / p o s)$ and recall $=1$, then

$$
p f=\alpha \cdot \frac{1-\text { prec }}{\text { prec }} .
$$

From (3), it is clear that for any targeted recall value, increasing precision requires decreasing false alarm rates; e.g., for prec $\in\{0.5,0.70,0.9,0.95\}$, pf becomes $\{1,0.43,0.11,0.005\}$, respectively. The effect is particularly marked for data sets with large neg/pos ratios (e.g., like the data processed by DMP).

\section{LARGE $\mathrm{Neg} / \mathrm{Pos}$ Ratios}

A detail not explored by the Zhangs is that many software engineering data sets have extremely large neg/pos ratios. For example:

- In the DMP paper, the data sets studied had neg/pos ratios of 1.04, 7.33, 9, 10.11, 13.29, 15.67, and 249.

- Hayes, Dekhtyar and Sundaram [9] use text mining to find pairs of connected requirements in a corpus of 220 requirements and 235 design elements (the same CM-1 data set used in [13]). The total number of possible links in the data set is $220 \cdot 235=51,700$, while the ground truth 


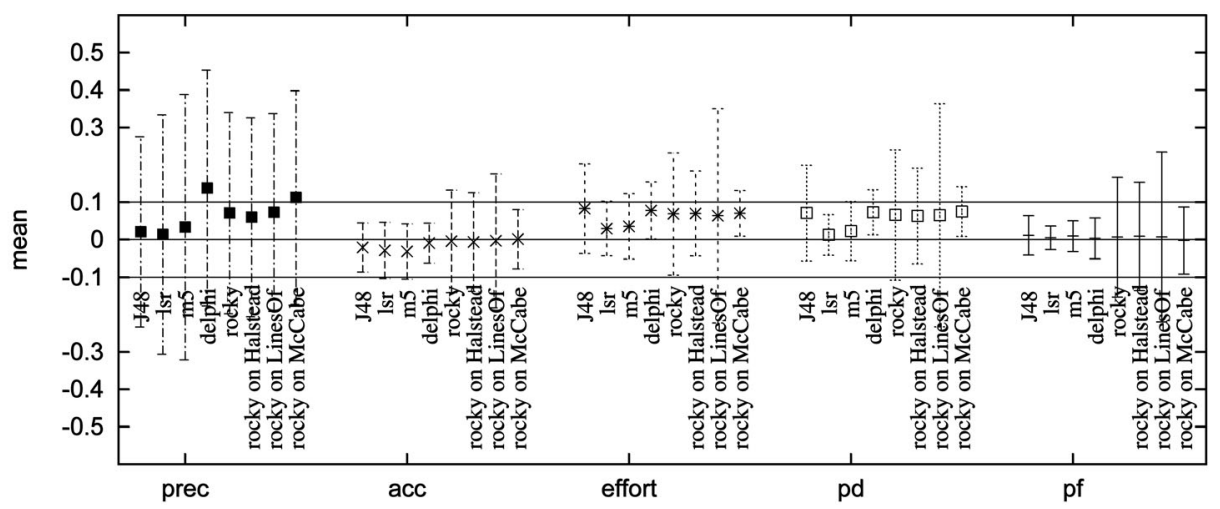

Fig. 1. Mean $\mu$ and standard deviation $\sigma$ of changes in defect detector statistics between a baseline (tested on the training set) and another data set tested on different data). A zero value denotes that the detector worked the same on training and test data. Dots denote mean $(\mu)$ values. Whiskers extend from $\mu+\sigma$ to $\mu-\sigma$. The data sets used in this study had some overlap with the DMP data, i.e., $c m 1, k c 1, k c 2, j m 1, p c$. This information was taken from [14].

RTM contains 361 links, for the neg/pos ratio of $51,700 / 361=143.2$.

- $\quad$ For an extreme example, Google reports that more than $10^{9}$ Web pages contain the phrase "software" but only one them is the home page of this journal. Hence, neg/pos for Web searching is at least $10^{9}$.

Fig. 2 graphs (1) for the DMP neg/pos ratios. Fig. 3 does the same, but is restricted to zones of higher precision: Only the surface for $0.5 \leq$ prec $\leq 1$ is shown. That shadow of the surface on the bottom plane shows that this is a zone of high precision, high recall, and large neg/pos. As neg/pos increases, high recall\&precision is only possible when $p f$ becomes vanishingly small. For example, in the ranges $0.65 \leq$ prec, recall $\leq 0.8$, (2) reports that $p f$ falls into the following ranges:

- $0.023 \leq p f \leq 0.062$ for $n e g /$ pos $=7$,

- $0.0108 \leq p f \leq 0.0287$ for $n e g / p o s=15$, and

- $\quad 0.007 \leq p f \leq 0.0017$ for $n e g / p o s=250$.

Detectors learned in the domain of software engineering rarely yield high precision detectors (see Fig. 4). Using the Zhangs' equations, the reasons for this are very clear:

- Those detectors all try to maximize recall.

- $\quad$ Fig. 2 shows that such detectors can only achieve high precision in the rare case of very low $p f$.

Not only does the Zhangs' equation explain the Fig. 4 results, it also informs the instability of precision and the stability of $p f$ and $p d$ (recall) seen in Fig. 1:
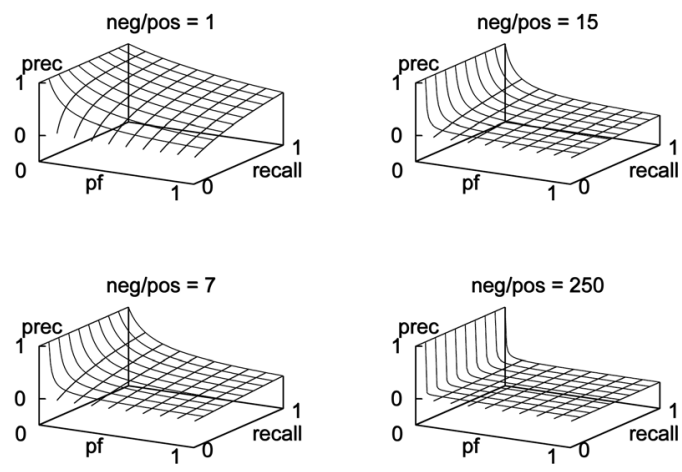

Fig. 2. The relationship between $p d$, prec, recall, and neg/pos.
- Note, in Fig. 2, that at very small $p f$ values, tiny changes in $p f$ can lead to very large changes in prec (sudden jumps from zero to one).

- The other measures in Fig. 2, on the other hand, change far more smoothly and slowly.

That is, the Zhangs' equation is the essential theoretical statement needed to explain numerous prior results such as those shown in Fig. 4 (i.e., [1], [2], [4], [9], [12], [13], [14]).

\section{When Low Precision Is Useful}

Achieving high precision\&recall can be problematic. As shown by the Zhangs' equation, optimizing for one often compromises the other (especially for data sets with large neg/pos ratios). Fortunately, there are many industrial situations where lowprecision and high-recall detectors are useful. For example, one of us (DiStefano) has used our low-precision detectors to review flight code developed at the NASA Glenn Research Center (Ohio). When the results of these detectors were presented to the lead flight engineer, he confirmed that the identified sections (which did not have any recorded defects) had been problematic to maintain and contained several bugs which had not yet been entered into the defect system.

For another example, from outside the field of SE, a user of a commercial Web search engine like Google can quickly flick through, say, three pages of results before finding a page of interest. Google has so many return customers since even with precisions of, say, $\frac{1}{30}$, the effort involved in looking at a page is so low that users don't mind examining 29 false alarms.
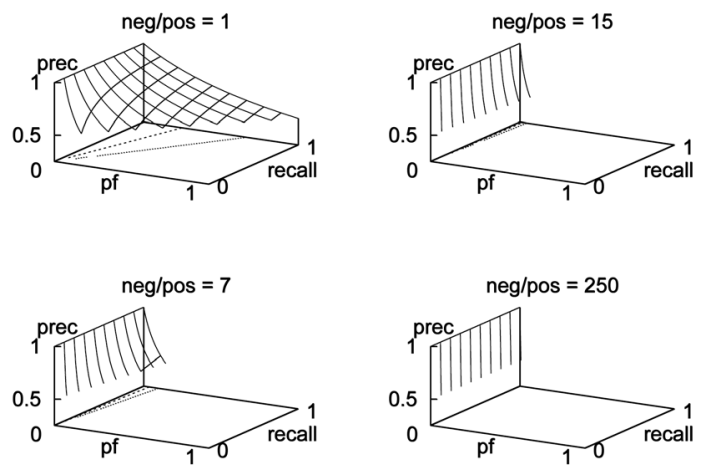

Fig. 3. Fig. 2, cropped to the region where prec $>0.5$. 
- The DMP paper achieved recalls over $70 \%$ and minimum false alarm rates of $15 \%$. Using the Zhangs' equation and data from DMP, it can be shown that the DMP precisions were quite low: $\{$ min, median, $\max \}=\{2 \% 20 \%, 70 \%\}$ (the last number came from a data set with neg/pos $\approx 1$ ).

- Huang et.al. [4] won "best paper" at the 2006 IEEE Requirements Engineering conference with detectors exhibiting prec $\approx$ 0.25 .

- Without extensive feedback from human experts, the classifiers used by Hayes, Dekhtyar, and Sundaram exhibited $p f>0.6$ (hence, very low precisions) [9].

- Other such as Antoniol et.al. [1], [2] and Marcus et.al. [12] researchers into software tracability report that high recall is achievable only with low precision detectors. Antoniol has also been exploring bug traces in Mozilla components and has found the same high-precision, low-recall trade-off.

- After much experiments with linear regression, model tree learners, Bayes classifiers, decision tree learners, singleton rule learners, and some home-brew learners [14], the general trend is very clear. For those learners, executing on the DMP datasets, $p f \leq p d-0.5$; That is, for those learners and those data sets, obtaining high recall figures of $p d>0.6$ implies $p f>0.1$ and, consequently, low precision.

Fig. 4. Some low precisions seen in the software engineering literature.

More generally, there are several situations where low precision detectors are useful:

- When the cost of missing the target is prohibitively expensive. In mission critical or security applications, the goal of 100 percent recall may be demanded in all situations, regardless of the precision.

- When only a small fraction of the data is returned. Hayes et al. call this fraction selectivity and offer an extensive discussion of the merits of this measure [9].

- When there is little or no cost in checking false alarms. For example, a detector we have found useful in industrial settings is to check modules where

$$
\frac{\text { lines of comments }}{\text { lines of code }}>0.25 \text {. }
$$

This detector triggers on complex functions that programmers comment extensively, instead of splitting up into smaller, more maintainable, functions. This detector is imprecise-it often triggers on well-written functions with detailed comments. However, based on commercial experience, we assert that it is fast and simple for a human agent to inspect the identified modules and discern which ones were well-written and which were overcommented to disguise being badly coded. We use this detector to find code that should be rewritten prior to release.

\section{RESeARCh DiRECTIONS}

Just because high precision\&recall detectors have not been seen before in SE does not mean that this goal is impossible. If large $n e g / p o s$ ratios are the problem, then perhaps the solution is to change those ratios in the training data. In "oversampling," the minority class (pos) is repeated multiple times. In "undersampling," some portion of the majority class (neg) is discarded. In this way, a training data set with $n e g / p o s=1$ might be generated. Fig. 2 shows that the space of neg/pos $=1$ detectors contains many candidates with both high precision\&recall. However, while a promising technique, there is contradictory evidence for its value.
Yun chung Liu reports that the appropriate resampling technique and appropriate classifier is data set dependent [3]. In limited studies with one learner and a few data sets, Drummond and Holte [6] found that oversampling had little value. They offer some evidence for the value of undersampling but concluded that other methods can do better. One issue is that, while resampling yields a training set that contains neg/pos $=1$, the test set still has the original distributions. In any case, this area is ripe for further exploration.

Another promising direction might be to try boosting. The AdaBoost algorithm [7] builds an ensemble of detectors $1,2,3, \ldots$. where detector $i$ is built from problems that were misclassified by detectors $1,2, \ldots, i-1$. AdaBoost defines a voting procedure for making conclusions after passing all new test instances to every member of the ensemble. It can be shown that increasing the size of the ensemble decreases error [7]. That is, the goal of both high precision\&recall might be achievable using boosting. Curiously, to do so, we require using detectors that generate enough false alarms to inform the boosting.

The theoretical advantage of boosting has yet to show significant improvements in real-world defect data sets (see the modest improvements of [10] or the poor comparative performance of AdaBoost compared to other methods in [11, pp. 64-77]). Therefore, to address the Zhangs' challenge of high precision\&recall, we need to look for other techniques.

Yet another avenue to explore is stacking, i.e., levering the strengths and weaknesses of different learners in an assembly that does better than any single learner. For example, Gaddam et. al. achieved high precision\&recall in one data set by combining clustering with decision tree learning. However, stacking is a poorly understood area and the behavior of the resulting assembly is difficult to predict. Gaddam et. al.'s toolkit only reached high precision\&recall in one data set; in several others, it could not [8].

Our final suggestion for how to achieve the Zhangs' goal is to augment automatic learning with some user modeling. Starting with unsupervised learners, it is possible to give learners information about the top candidate conclusions. A feedback loop can then be entered as a kind of iterative supervised learning (results from generation $i$ inform and improve the results at generation $i+1)$. This methodology leads to two different things you can measure: 1 ) the recall/precision of the current output, or 2) the recall/precision of the list of candidate conclusions used for learning during multiple iterations:

- In [9], the former is measured and Hayes et al. iteratively refine their learned detectors, transforming low-precision detectors into high-precision detectors. In one case study, the reached precisions and recalls increased more than 0.85 (in the current output) after five rounds of users reviewing and commenting on the learned detectors. The challenge with this method is that is requires extensive involvement by knowledgeable users - and such users can be a scarce and expensive resource. Researchers exploring this approach must balance the benefits of high precision\&recall detectors against their high construction cost.

- In subsequent work [5], Dekhytar et al. measured the recall/precision of the candidates used during multiple iterations. They found that they were still faced with the same low-precision/high-recall trade-off, e.g., to have seen 90 percent of the correct candidates, we had to go through a list whose precision is about 20 percent.

In summary, while the above techniques show promise, it may take much further research to achieve high precision\&recall detectors in SE data sets with large neg/pos ratios. 


\section{Conclusion}

The Zhangs argue that predictors are useless unless they have high precison\&recall. We have a different view, for two reasons. First, for SE data sets with large neg/pos ratios, it is often required to lower precision to achieve higher recall. Second, there are many domains where low precision detectors are useful.

Nevertheless, there is much value in Zhangs' equation. It is a useful result that explains numerous prior results such as [1], [2], [4], [9], [12], [13], [14]. Zhang and Zhang's equation also explains why precision is much less stable than other measures. Hence, researchers are advised not to use precision when assessing their detectors. Other measures are more stable (i.e., recall $(p d)$ and false alarm rates), especially for data sets with large neg/pos ratios.

\section{References}

[1] G. Antoniol, G. Canfora, G. Casazza, A. De Lucia, and E. Merlo, "Recovering Traceability Links between Code and Documentation," IEEE Trans. Software Eng., vol. 28, no. 10, pp. 970-983, Oct. 2002.

[2] G. Antoniol and Y.-G. Gueheneuc, "Feature Identification: A Novel Approach and a Case Study," Proc. Int'l Conf. Software Maintenance (ICSM '05) pp. 357-366, 2005.

[3] A. Yun chung Liu, "The Effect of Oversampling and Undersampling on Classifying Imbalanced Text Datasets," master's thesis, http://www.lans. ece.utexas.edu/aliu/papers/aliu_masters_thesis.pdf, 2004.

[4] J. Cleland-Huang, R. Settimi, X. Zou, and P. Solc, "The Detection and Classification of Non-Functional Requirements with Application to Early Aspects," Proc. Requirements Eng. Conf. (RE'06), pp. 36-45, 2006.

[5] A. Dekhtyar, J.H. Hayes, and J. Larsen, "Make the Most of Your Time: How Should the Analyst Work with Automated Traceability Tools?" Proc Third Int'l Workshop Predictive Modeling in Software Eng. (PROMISE '07), 2007.

[6] C. Drummond and R.C. Holte, "C4.5, Class Imbalance, and Cost Sensitivity: Why Under-Sampling Beats Over-Sampling," Proc. Workshop Learning from Imbalanced Datasets II, 2003.

[7] Y. Freund and R.E. Schapire, "A Decision-Theoretic Generalization of Online Learning and an Application to Boosting," J. Computer and System Sciences, vol. 55, 1997.

[8] S.R. Gaddam, V.V. Phoha, and K.S. Balagani, "K-means+id3: A Novel Method for Supervised Anomaly Detection by Cascading k-Means Clustering and id3 Decision Tree Learning Methods," IEEE Trans. Knowledge and Data Eng., vol. 19, no. 3, Mar. 2007.

[9] J.H. Hayes, A. Dekhtyar, and S.K. Sundaram, "Advancing Candidate Link Generation for Requirements Tracing: The Study of Methods," IEEE Trans. Software Eng., vol. 32, no. 1, Jan. 2006.

[10] T.M. Khoshgoftaar, E. Geleyn, L. Nguyen, and L. Bullard, “Cost-Sensitive Boosting in Software Quality Modeling," Proc. Symp. High Assurance Software Eng., p. 51, 2002.

[11] Y. Ma, "An Empirical Investigation of Tree Ensembles in Biometrics and Bioinformatics," PhD thesis, Jan. 2007.

[12] A. Marcus and J. Maletic, "Recovering Documentation-to-Source Code Traceability Links Using Latent Semantic Indexing," Proc. 25th Int'l Conf. Software Eng., 2003.

[13] T. Menzies, J. Greenwald, and A. Frank, "Data Mining Static Code Attributes to Learn Defect Predictors," IEEE Trans. Software Eng., vol. 33, no. 1, pp. 2-13, Jan. 2007.

[14] T. Menzies and J.S. Di Stefano, "How Good Is Your Blind Spot Sampling Policy?" Proc. IEEE Conf. High Assurance Software Eng., http://menzies.us/ pdf/03blind.pdf, 2003.

[15] H. Zhang and X. Zhang, "Comments on 'Data Mining Static Code Attributes to Learn Defect Predictors,'" IEEE Trans. Software Eng., Sept. 2007. 